\title{
Cysteine Proteinases and Their Inhibitors - A Traditional Topic of the Portoroz Conferences
}

This issue of Biological Chemistry focuses on structural and functional aspects of cysteine proteinases and their inhibitors, the topic of the recent meeting in Portoroz/Slovenia (September 9-13, 2000) organized by Vito Turk, his sons, and colleagues from the Jozef Stefan Institute in Ljubljana/Slovenia. It is dedicated to a scientist who has been successfully engaged in this area for a long time but prefers to do his work quietly, primarily at the bench of his lab amidst his team: Professor Werner Machleidt from the Ludwig-Maximilians-University, Munich, Germany, on the occasion of his $60^{\text {th }}$ birthday.

I (Hans Fritz) became acquainted with his creative and subtle style first in 1968 when Theodor Bücher brought us together in the Sonderforschungsbereich SFB 51 'Biochemistry and Molecular Biology in Munich', a special research unit at the LMU which is peer-reviewed and financed by the Deutsche Forschungsgemeinschaft. Since that time his projects have continously been supported by SFBs, unequivocally proving his scientific reputation.

In 1964, as a young medical student, Werner Machleidt asked Professor Theodor Bücher for a doctoral thesis and was assigned to develop a high-sensitivity amino acid analyzer. This was the onset of a persistent scientific passion devoted to the establishment of novel methods and their application to the structural and functional analysis of proteins. At that time only few proteins had been sequenced because amino acid sequencing was a complicated and tedious manual procedure. Taking advantage of Theodor Bücher's excellent mechanical workshop, Werner Machleidt developed the first fully automated solid-phase sequencer operating on-line with HPLC, a principle that has become the standard in todays gas phase sequencers. The structures of many interesting proteins were sequenced in his laboratory, among them the Rieske iron-sulfur protein of the $\mathrm{bc}_{1}$ complex and the human cathepsins $\mathrm{B}, \mathrm{H}$, and $\mathrm{L}$. Amino acid sequencing of several cysteine proteinase inhibitors that had been isolated in Vito Turk's laboratory led to the discovery of the superfamily of cystatins. Together with Werner Müller-Esterl, it was shown in Werner Machleidt's group that the kininogens function as cysteine proteinase inhibitors.

In the middle of the 80 's, when protein sequencing was rapidly superseded by DNA sequencing, Werner Machleidt began to study the functions of cysteine proteinases and their inhibitors. Again he started with improvements of the existing methodology of enzyme/inhibition kinetics. He introduced sensitive continuous assays with online digital data collection that allow the precise characterisation of tight-binding inhibitors. Work using recombinant cystatin variants produced by Ennes Auerswald in Hans Fritz' laboratory confirmed and extended the mechanisms of inhibition postulated from the crystal structures of cysteine proteinases and cystatins obtained in Wolfram Bode's group. Together with Marianne Jochum, cathepsin B was identified as an important marker of inflammation in clinical studies of polytrauma and peritonitis patients. Synthetic inhibitors and affinity labels directed against pericellular cathepsin B of tumor cells have been designed and evaluated together with Louis Moroder's group. Recently, the surface plasmon resonance technology (BIACORE) has successfully been utilized to study proteinase-inhibitor interactions.

Currently, Werner Machleidt leads an active research group working on calpains and their protein inhibitors, calpastatin and kininogen. His research topics range from structure-function relationships and molecular mechanisms to the cellular localization of the calpain/calpastatin system and its role in apoptosis.

Hans Fritz and Marianne Jochum

Department of Clinical Chemistry

and Clinical Biochemisty

Ludwig-Maximilians-University

Nussbaumstrasse 20

D-80336 Munich, Germany 